\title{
Quiet Sun mini-coronal mass ejections activated by supergranular flows ${ }^{\star}$
}

\author{
D. E. Innes ${ }^{1}$, A. Genetelli ${ }^{1,2}$, R. Attie ${ }^{1}$, and H. E. Potts ${ }^{3}$ \\ 1 Max-Planck Institut für Sonnensystemforschung, 37191 Katlenburg-Lindau, Germany \\ e-mail: innes@mps.mpg.de \\ 2 Université Paul Sabatier, 31062 Toulouse, France \\ 3 Department of Physics and Astronomy, University of Glasgow, Glasgow G12 8QQ, UK
}

Received 22 September 2008 / Accepted 21 November 2008

\section{ABSTRACT}

\begin{abstract}
Context. The atmosphere of the quiet Sun is controlled by photospheric flows sweeping up concentrations of mixed polarity magnetic field. Along supergranule boundaries and junctions, there is a strong correlation between magnetic flux and bright chromospheric and transition region emission.

Aims. The aim is to investigate the relationship between photospheric flows and small flare-like brightenings seen in Extreme Ultraviolet images.

Methods. We describe observations of small eruptions seen in quiet Sun images taken with the Extreme UltraViolet Imager (EUVI) on STEREO. The photospheric flows during the eruption build-up phase are investigated by tracking granules in high resolution MDI continuum images.

Results. Eruptions with characteristics of small coronal mass ejections (CMEs) occur at the junctions of supergranular cells. The eruptions produce brightening at the onset site, dark cloud or small filament ejections, and faint waves moving with plane-of-sky speeds up to $100 \mathrm{~km} \mathrm{~s}^{-1}$. In the two examples studied, they appear to be activated by converging and rotating supergranular flows, twisting small concentrations of opposite polarity magnetic field. An estimate of the occurrence rate is about 1400 events per day over the whole Sun. One third of these events seem to be associated with waves. Typically, the waves last for about 30 min and travel a distance of $80 \mathrm{Mm}$, so at any one time they cover 1/50th of the lower corona.
\end{abstract}

Key words. Sun: activity - Sun: UV radiation - Sun: coronal mass ejections (CMEs)

\section{Introduction}

Coronal mass ejections (CMEs) are large-scale eruptions of plasma into the solar corona. In coronagraph images, a classical CME has a three-part structure. There is a fast outer front with a large dark cavity behind and inside a bright dense core. Against the disk, extreme ultraviolet (EUV) images usually detect a flarelike brightening at the onset site, a compact dark eruption, and sometimes a dimming propagating outwards across the disk. CMEs are thought to be triggered by instability leading to magnetic reconnection, and brought on by convective flows at the footpoints of large magnetic loops. Mini-CMEs have the same characteristics on a smaller scale. They will be most easily identified against the quiet Sun because in active regions they disappear into the general background activity and off-limb there is too much line-of-sight confusion.

Mini-CMEs have not, as such, been reported previously; however it is likely that parts of them have. The small erupting filaments seen by Hermans \& Martin (1986) and Wang et al. (2000) with a typical lifetime of $50 \mathrm{~min}$, speed of $13 \mathrm{~km} \mathrm{~s}^{-1}$, and length of $20^{\prime \prime}$, may be the erupting core. If so, mini-CMEs could be expected to have the same high rate of about 6000 events per $24 \mathrm{~h}$ on the Sun (Wang et al. 2000). They may also be related to the flare-like brightenings seen in high cadence TRACE $171 \AA$ quiet Sun images discussed by Ireland et al. (1999). In that sequence, the TRACE exposure time was probably too short to

$\star$ Movies are only available in electronic form at http://www. aanda.org detect mini-filament eruptions as darkenings in the EUV, and the analysis only describes brightenings.

High signal-to-noise observations are now available at cadences fast enough to see both mini-filament eruptions and brightenings from the recently launched EUV Imagers (EUVI, Howard et al. 2008) on the two STEREO spacecraft. The two spacecraft have slightly different orbits. One is inside the Earth (Ahead) and the other outside (Behind) so that the spacecraft are gradually moving apart in order to observe the Sun simultaneously from different angles. At the time of the observations analysed here, the STEREO spacecraft were 12 degrees apart, and each about 6 degrees from the Earth. In principle, the two images can be combined to reconstruct the 3-D structure of events (Feng et al. 2007; Aschwanden et al. 2008). When they are near 90 degrees apart it will be possible to analyse both the on-disk and off-limb view of (mini-)CMEs.

One advantage of the small angle is that it allowed us to simultaneously observe the photospheric magnetic field and flows in high resolution with MDI/SoHO, and test the importance of supergranulation flows during the pre-eruption phase. Convective flows below the solar surface sweep up small concentrations of mixed-polarity magnetic field into the distinctive chromospheric network pattern of supergranular cells (Martin 1988; Wang 1988; Schrijver et al. 1997; Hagenaar 2001; Parnell 2002). The chromospheric emission and activity is strongest along the boundaries where the magnetic flux interactions occur (Porter et al. 1987; Moore et al. 1999; Dere et al. 1991; Aiouaz 2008). Models of the energy released during interaction have 
been suggested by several authors (Priest et al. 1994; Moore et al. 1999; Longcope \& Kankelborg 1999; Priest et al. 2002). In these models magnetic energy is converted into plasma heat and kinetic energy at reconnection sites, producing hot loops and plasma jets. Another aspect discussed by Priest et al. (1994), Moore (1988) and Moore et al. (1999), and central to the observations discussed here, is the formation and eruption of filaments above the interacting field regions. Filaments tend to form along magnetic neutral lines with a large degree of magnetic twist or shear (Priest et al. 1989; van Ballegooijen \& Martens 1989; van Ballegooijen et al. 2000; Amari et al. 2000). The twist can be measured from vector magnetograms, and it is caused by rotation and movement of the photospheric footpoints.

Most UV and EUV quiet Sun dynamic events appear to be small scale microflares or X-ray bright points that heat the plasma locally. To contribute to coronal heating the energy must be carried into the corona. Waves generated by impulsive events have so far only been unambiguously identified in large CMEs and flares (Moreton \& Ramsey 1960; Thompson et al. 1998; Khan \& Aurass 2002; Biesecker et al. 2002; Warmuth et al. 2004), and these are not frequent enough to heat the whole corona. If, however, waves are associated with mini-CMEs and they have a rate comparable to that of mini-filament eruptions, then they may be a viable mechanism for heating the corona.

In this paper, we show features of small CME-like eruption events occurring at the junctions of supergranular cells. The EUV images show the flaring hot plasma through a brightness increase, plasma evacuations due to waves by a dimming, and cold plasma ejections as a darkening. We demonstrate with examples of space-time intensity variations, the frequency and general characteristics of the events. When tracking the photospheric flows and magnetic field in two events, we find vortex-like structure at the supergranular junction preceding the eruptions.

\section{Data preparation}

The region chosen for study (Fig. 1) included a small equatorial coronal hole at disk center with active regions on either side, and a large area of quiet Sun to the north. It was selected because it contained a variety of different solar structures passing disk center at a time when both synoptic EUVI/STEREO $171 \AA$ and an $8 \mathrm{~h}$ series of MDI high resolution data were available.

EUVI/STEREO $171 \AA$ synoptic images of the full Sun were taken on 2007 June 11 with the two STEREO spacecraft (Ahead and Behind) throughout the day with a cadence of $2.5 \mathrm{~min}$ and pixel size of $1.59^{\prime \prime}$. Here only images from the Ahead spacecraft are discussed. We have used the Behind images to confirm faint structures seen in the Ahead images. There were $586 \mathrm{im}-$ ages from each spacecraft, and 195 of these overlapped with the time of high resolution MDI observations. The EUVI images were calibrated and rotated to the SoHO viewpoint at the time of the first high resolution MDI image (07:51 UT) using standard routines available in SolarSoft.

MDI high resolution (pixel size $0.625^{\prime \prime}$ ) observations were made from 07:51 UT to 16:06 UT with a cadence of one frame per min, over an area $622^{\prime \prime} \times 304^{\prime \prime}$. The continuum and magnetic field images were de-rotated to the eastern $516^{\prime \prime}$ of the 07:51 UT image using SolarSoft routines. The continuum images were used to compute photospheric horizontal flows using the Balltrack method described by Potts et al. (2004, 2007).

The coalignment between EUVI and MDI was checked by comparing MDI full disk with the full disk EUVI images. Since

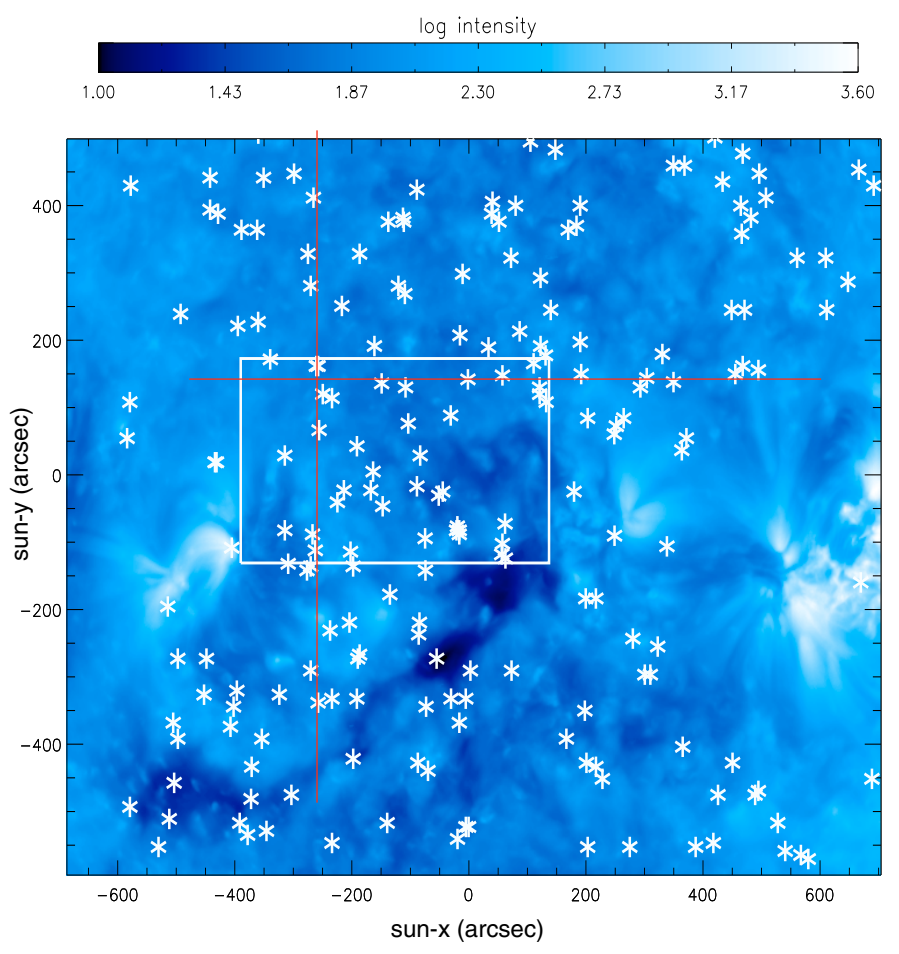

Fig. 1. The average $171 \AA$ intensity image for the region studied on 11 June 2007, with events and the region of high resolution MDI marked. The horizontal and vertical red lines indicate the positions of the spacetime images shown in Fig. 2.

there were the two active regions and the limb to coalign with, we believe the coalignment to be accurate to $4^{\prime \prime}$ (two full disk MDI pixels).

\section{Eruption events}

Eruption events are seen with a fairly wide range of characteristics in the EUV images. The basic feature is a brightening at the eruption site and nearly always a small, dark plasmoid ejection. In stronger events there is, in addition, a faint wave-like feature that travels several tens of arcsec from the source. For this initial study, we have used a very simple event identification method based on recognizing emission or absorption trains by eye in series of time-distance $171 \AA$ images. Examples of time-distance images are shown in Fig. 2. These pictures show many abrupt changes in the EUV emission and we have had to make sensible choices for selecting events. On the computer screen events show up more clearly, especially the faint wave-like extensions. The first thing we did was run through the dataset displaying spacetime images row-by-row. If an event was seen in any image, its space-time co-ordinates were filed. Afterwards only events seen over 6 " (3 consecutive pixels) were kept. Then any events closer than $40^{\prime \prime}$ in space and $50 \mathrm{~min}$ in time to any other recorded event were removed. This made sure that the same event was not counted twice. The event positions, marked as asterisks in Fig. 1, are those that remain. Because there is no check on the event strength seen at a particular position, the event positions (asterisks) are often 10"-20" from the event center. Almost no events have been recorded near the active region loop systems due to the confusion with loop brightenings.

In Fig. 2, registered events have been circled. Here a variety of features have been circled because different parts of events are crossing these particular space-time slices. The larger ones are numbered and the measured lifetimes and velocities in the 


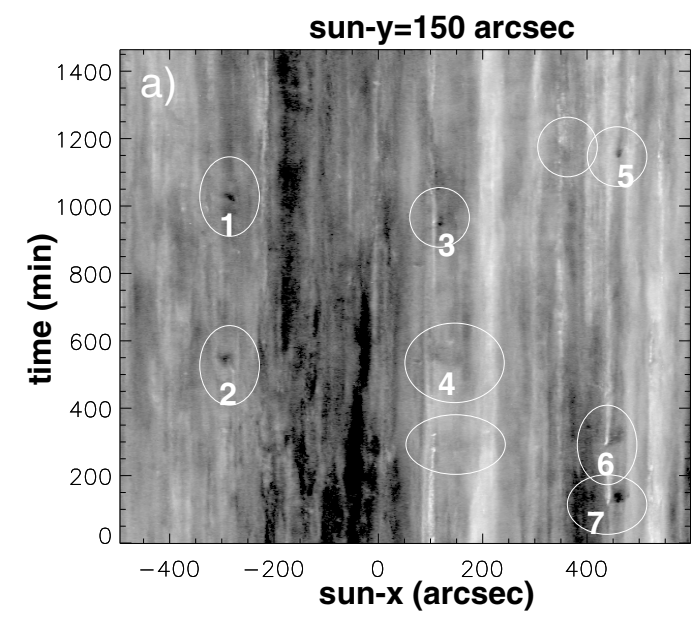

Table 1. Velocity and lifetime of dark ejections and faint waves in Figs. 2 and 3.

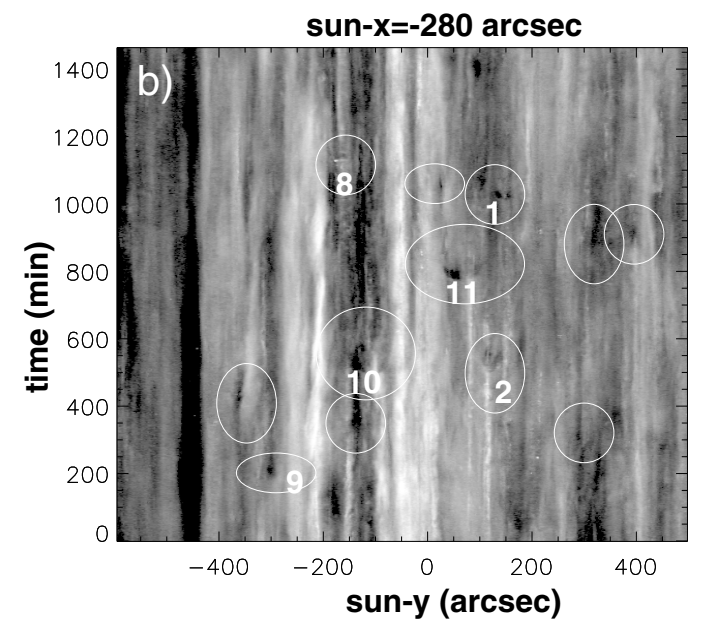

Fig. 2. Time series of EUVI $171 \AA$ A images for slices of quiet Sun along the sun- $x$ and sun- $y$ directions (see Fig. 1). Events are circled. The slices cross near events 1 and 2 so these events are seen in both images. The numbered events are shown in more detail in Fig. 3.

sun- $x$ direction of the dark and wave-like features are given in Table 1. More detailed images of the individual events are shown in Fig. 3, where dotted lines are drawn along the edge of the faint waves. These are sometimes curved because the waves slow down. In these cases the velocity in the Table is the average velocity. The lifetimes are minimum event lifetimes, since they were measured from the displayed space-time images, and in a different direction the lifetime may appear longer. As can be seen in Fig. 2, for almost one third of the events it is not possible to measure velocities. There are several darkenings that could be events, but have not been included. This makes the statistics rather uncertain. In the future we hope to develop a more quantitative scheme.

The $1200^{\prime \prime} \times 1100^{\prime \prime}$ area studied is mostly quiet Sun. It covers about $1 / 8$ th of the solar surface. In this region, 176 events were recorded in $24 \mathrm{~h}$. This gives a rate of 1 event per min or 1400 per day over the whole Sun, which is about a quarter of the rate of quiet Sun $\mathrm{H} \alpha$ mini-filament eruptions found by Wang et al. (2000). The typical lifetimes and velocities of the dark ejecta are very similar to the $\mathrm{H} \alpha$ mini-filaments so we believe these are the same phenomena. The numbers in Table 1 indicate that the wave-like features have an average velocity $\sim 45 \mathrm{~km} \mathrm{~s}^{-1}$, and lifetime $\sim 30 \mathrm{~min}$. Therefore the distance travelled is approximately $80 \mathrm{Mm}$. Assuming the statistics of Fig. 2 are typical, one third of events have detectable waves. This corresponds to $1 / 50$ th of the lower corona being affected by these events at any one time.

\begin{tabular}{cccc}
\hline \hline Event & Feature & $V\left(\mathrm{~km} \mathrm{~s}^{-1}\right)$ & Lifetime $(\mathrm{min})$ \\
\hline 1 & dark & 14 & 25 \\
2 & dark & 6 & 30 \\
& wave & 26 & 40 \\
3 & dark & 21 & 20 \\
4 & wave & 41 & 19 \\
5 & dark & 5 & 20 \\
6 & dark & 19 & 45 \\
& wave & 36 & 13 \\
7 & dark & 16 & 50 \\
& wave & 24 & 30 \\
8 & wave & 100 & 6 \\
9 & dark & 18 & 40 \\
10 & dark & 15 & 50 \\
& wave & 50 & 30 \\
11 & dark & 26 & 42 \\
& wave & 36 & 60 \\
\hline
\end{tabular}

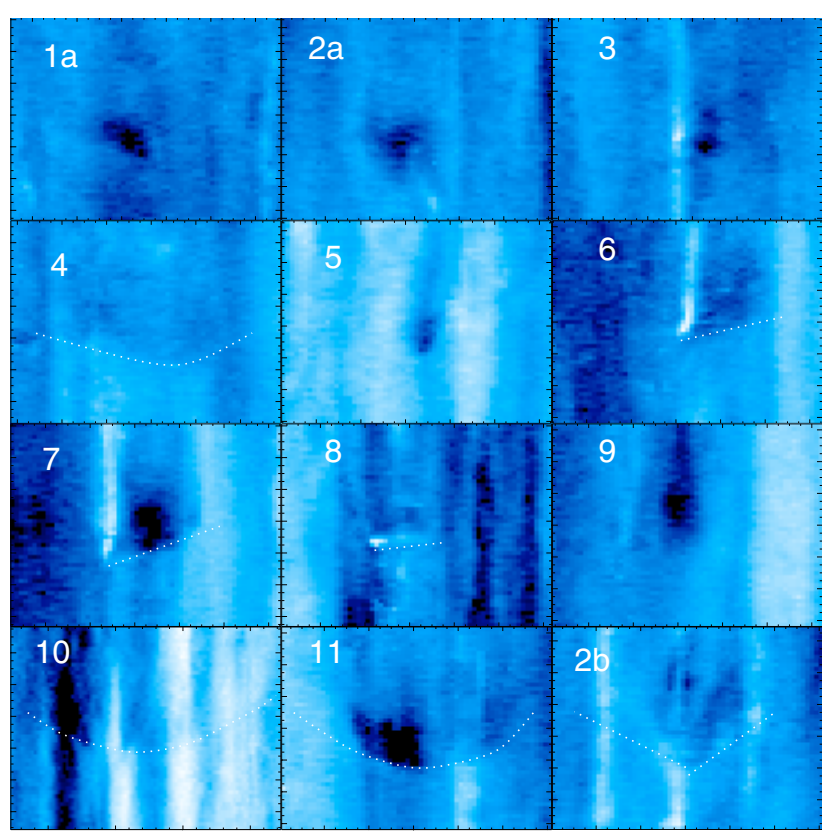

Fig. 3. Close-up of events numbered in Fig. 2. The plane-of-sky velocities and lifetimes of the faint waves and dark features are given in Table 1. White dotted lines trace the edges of the wave-like features. The frames are $100^{\prime \prime}$ by $125 \mathrm{~min}$, except for event 10 which is $160^{\prime \prime}$ by 125 min.

\section{Photospheric flows}

The activity of the corona is strongly coupled to the supergranular photospheric flows (Potts et al. 2007). It is well known that supergranular lanes and the junctions of supergranular cells are associated with transition region activity. Coupling the flows to the coronal emission was therefore a major goal of this work.

After the basic flows have been computed using the Balltrack method with MDI continuum images (Potts et al. 2004), we represent the photospheric transverse motion as arrows that move with the flow. New arrows are continually added at random positions where there are few arrows and removed from regions where they cluster together. Arrows, acting like corks, collect at supergranular cell boundaries and junctions, and disappear from cell centers.

For a single flow field, the arrows are integrated until a converged solution for that flow field is reached. This representation 


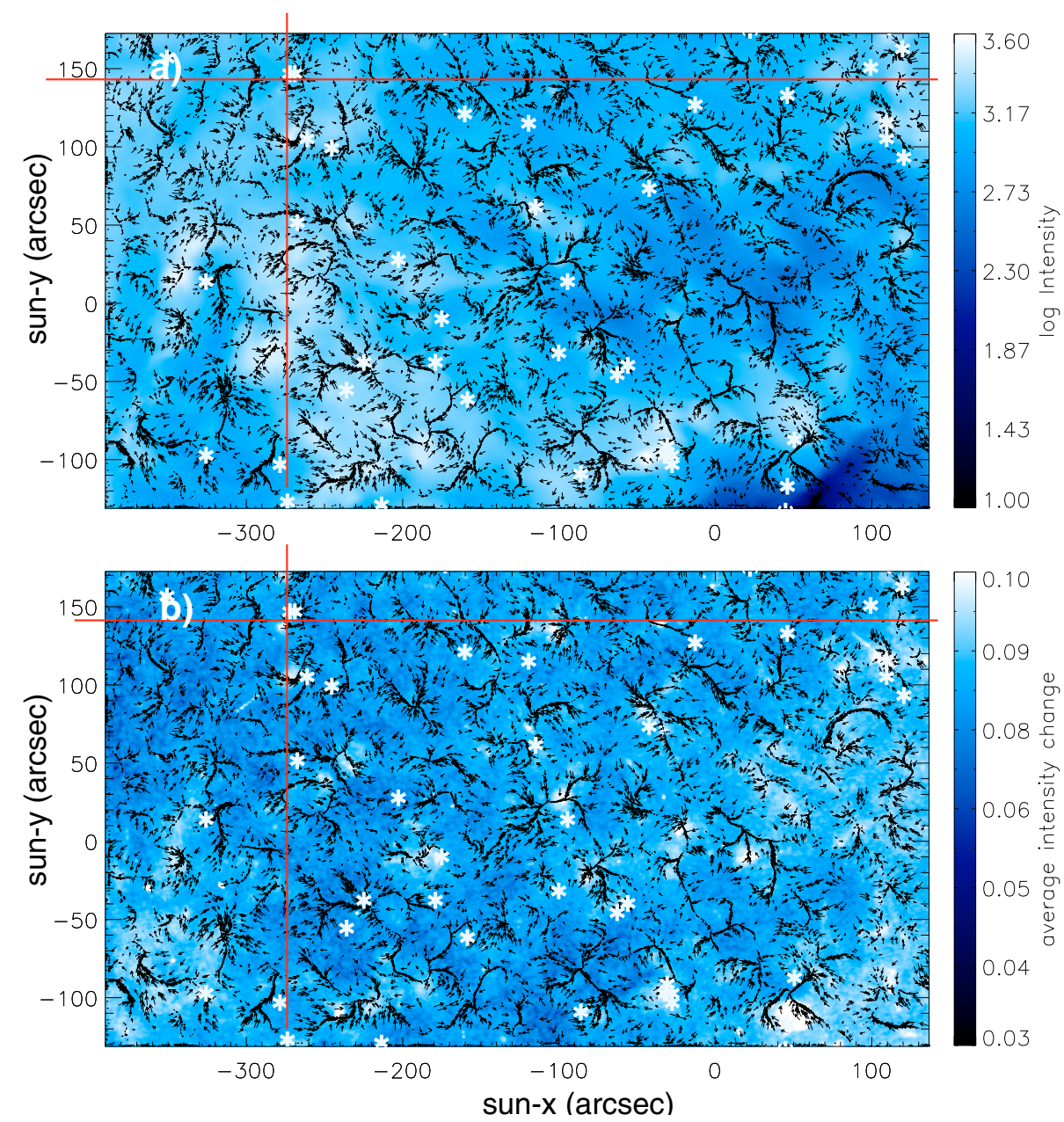

Fig. 4. The relationship between photospheric flows, EUV intensity and brightenings, and the positions of mini-CME events. a) The average $171 \AA$ intensity for the region studied on 11 June 2007. b) The average intensity change over $2.5 \mathrm{~min}$ (the time between images) normalized by the average intensity. The photospheric flows are represented as black arrows that accumulate along the cell boundaries. The white asterisks indicate approximate positions $\left( \pm 20^{\prime \prime}\right)$ of mini-CME events. The horizontal and vertical lines indicate the positions of the space-time images in Fig. 2. of the average flow field over the $8 \mathrm{~h}$ MDI period is shown in Fig. 4, superimposed on the average $171 \AA$ intensity and the normalized average intensity change over $2.5 \mathrm{~min}$. The normalized average intensity change is the average of the running difference images over the $8 \mathrm{~h}$ period, divided by the average intensity. When a time series of photospheric flows is available, then the arrow positions respond to the changing flow field, so there is a small lag in the arrow positions compared with the current flow field. For the discussion here, where we are considering properties on the scale of supergranular cells, this lag is insignificant. It ensures however that the arrows in the accompanying animations follow smoothly from one frame to the next.

In Fig. 4, the positions of events are shown as white asterisks. Events 1 and 2 in Fig. 2 are the two almost superimposed asterisks near $(-270,150)$. The lower image shows that nearly all rapid intensity changes occur, as expected, along supergranular boundaries, where the flows (represented by black arrows) accumulate. The biggest brightening occurred on the coronal hole boundary at $(50,-120)$, where there were two strong events. Both were seen near the beginning of the $8 \mathrm{~h}$ period so it was not possible to see the photospheric flows in the build-up phase of these events. In the next section we show the build-up and eruption of two other events.

\subsection{Events}

As stated previously, there is a large variety of quiet Sun miniCME events, just like there is a large variety of flares and CMEs. The characteristics are probably dependent on structure of the event surroundings. In almost all cases both a brightening and a dark plasmoid eruption were seen. Here we show two events. One is a small dark cloud eruption and the other a flare-like event. Both were unique in their large-scale properties.

The first, shown in Fig. 5 and in the animation cloud_CME.gif, is the cloud-like eruption. Even in the quiet Sun there is a lot of activity and events like these that appear as a darkening, are difficult to detect. We have therefore put a white box around the event in the figure. The eruption occurred from the outlined supergranular junction and extended to the region of positive field on the right. The vortex in the photospheric flows at the supergranulation junction is well established at the time of the eruption. After the dark cloud eruption, the region between the opposite polarity fields brightened. It is interesting to note that the dark cloud seemed to stop at the supergranular boundary to the south before fading into the background.

The other event (Fig. 6 and in the animations flare_CME.gif and wave_CME.gif), was a more spectacular quiet Sun mini-CME. It showed brightening, filament eruption and wave-like dimming. The rotation seen in the photospheric flows, at the supergranular cell junction with opposite polarity fields, started about three hours before the eruption. After approximately $1.5 \mathrm{~h}$ the flows changed quite significantly, producing a sharp lane flowing eastward from the junction. It may have been this strong stretching of magnetic footpoints that triggered the eruption. The event occurred on the edge of the MDI field-of-view, so we were not able to trace the wave propagation in relation to the supergranulation field structure. We show the wave in the accompanying series of 


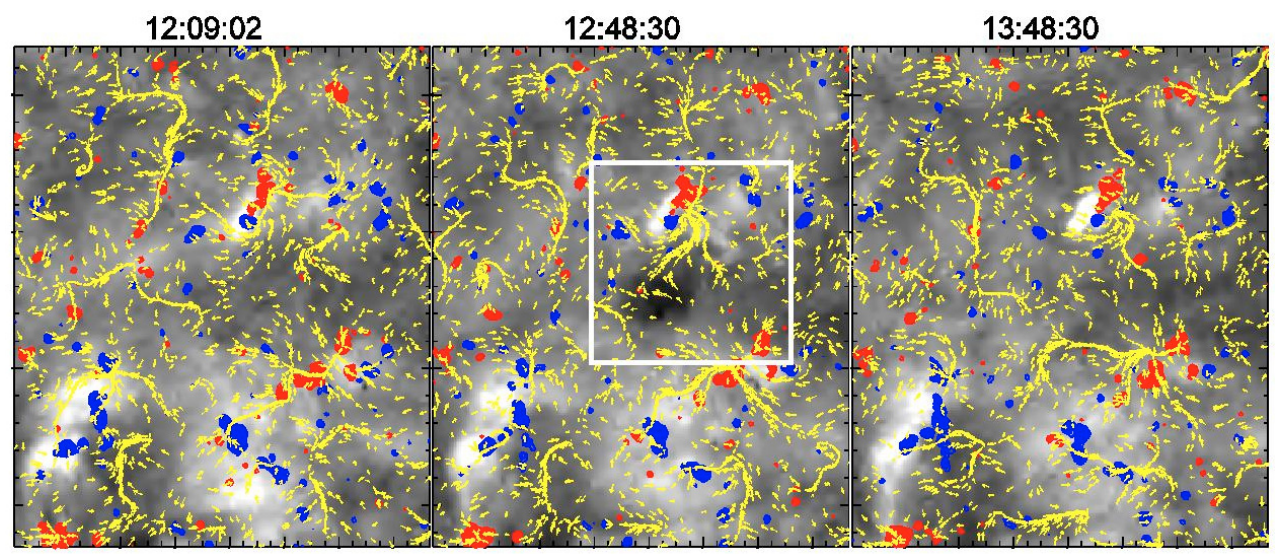

Fig. 5. A cloud-like mini-CME. STEREO 171 images (greyscale) with regions of magnetic field greater (less) than $40 \mathrm{G}(-40 \mathrm{G})$ coloured in blue (red), and the photospheric flows represented with yellow arrows. The FOV is $150^{\prime \prime} \times 180^{\prime \prime}$, centered at $(-285$, 78). The white box outlines the event. A movie of the temporal evolution of the photospheric flows, magnetic field, and EUV intensity is shown in cloud_CME.gif.

13:23:30

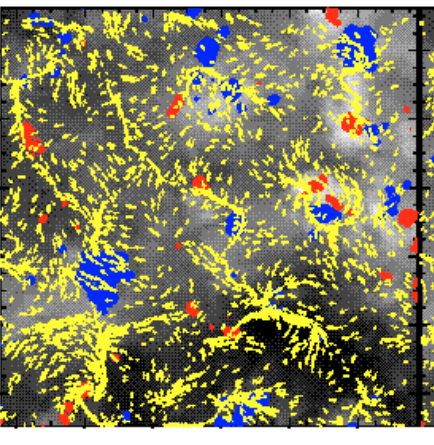

15:26:00

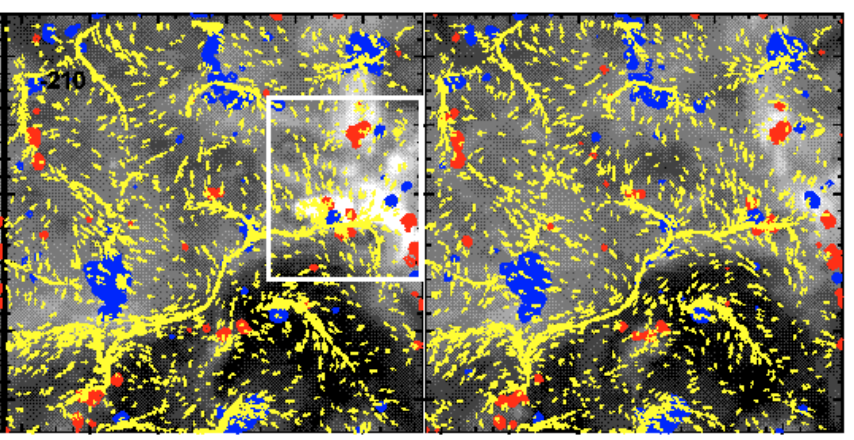

Fig. 6. A flare-like mini-CME. The representation is the same as Fig. 5 for FOV $125^{\prime \prime} \times 125^{\prime \prime}$, centered at $(75$, 110). A movie of the photospheric flow, magnetic field, and EUV intensity is shown in flare_CME.gif, and of the EUV alone over a larger FOV in wave_CME.gif.

$171 \AA$ images, wave_CME.gif, moving very quickly to the top right corner. The field-of-view in the animation is $150^{\prime \prime} \times 150^{\prime \prime}$.

\section{Discussion}

In this paper we have presented a preliminary overview of what we call mini-CMEs in the quiet Sun. Most of the events found show both mini-filament eruption and microflare brightening, and in several events wave-like features are seen propagating from the eruption site. We have shown that in the two cases studied the eruptions are driven by sheared network core fields. The natural question is whether these eruptions are responsible for coronal heating in the quiet Sun as proposed by Moore et al. (1999). The event rate is 1400 per day over the whole Sun, but only one third of these have observable wave-like fronts. If one assumes a typical lifetime of $30 \mathrm{~min}$ and velocity of $45 \mathrm{~km} \mathrm{~s}^{-1}$, they are still too infrequent and small to affect a significant area of the lower corona. Future observations from the solar Dynamics observatory (SDO) should provide far superior images over a wider range of temperatures as well as complementary photospheric fields and flows, and analysis of these images may provide further clues to how energy is transferred to the corona.

Acknowledgements. We would like thank the referee for very constructive comments. The data were provided by the MDI/SOHO and EUVI/STEREO consortia. STEREO is a project of NASA, SOHO a joint ESA/NASA project. The EUVI data used here were produced by an international consortium of the Naval Research Laboratory (USA), Lockheed Martin Solar and Astrophysics Lab (USA), NASA Goddard Space Flight Center (USA), Rutherford Appleton Laboratory (UK), University of Birmingham (UK), Max-Planck-Institut for Solar System Research (Germany), Centre Spatiale de Liège (Belgium), Institut d'Optique Théorique et Appliqueé (France), and Institut d'Astrophysique Spatiale (France).

\section{References}

Aiouaz, T. 2008, ApJ, 674, 1144

Amari, T., Luciani, J. F., Mikic, Z., \& Linker, J. 2000, ApJ, 529, L49

Aschwanden, M. J., Burlaga, L. F., Kaiser, M. L., et al. 2008, Space Sci. Rev., 136,565

Biesecker, D. A., Myers, D. C., Thompson, B. J., Hammer, D. M., \& Vourlidas, A. 2002, ApJ, 569, 1009

Dere, K. P., Bartoe, J.-D. F., Brueckner, G. E., Ewing, J., \& Lund, P. 1991, J. Geophys. Res., 96, 9399

Feng, L., Inhester, B., Solanki, S. K., et al. 2007, ApJ, 671, L205

Hagenaar, H. J. 2001, ApJ, 555, 448

Hermans, L. M., \& Martin, S. F. 1986, in Coronal and Prominence Plasmas, ed. A. I. Poland, 369

Howard, R. A., Moses, J. D., Vourlidas, A., et al. 2008, Space Sci. Rev. 136, 67 Ireland, J., Wills-Davey, M., \& Walsh, R. W. 1999, Sol. Phys., 190, 207

Khan, J. I., \& Aurass, H. 2002, A\&A, 383, 1018

Longcope, D. W., \& Kankelborg, C. C. 1999, ApJ, 524, 483

Martin, S. F. 1988, Sol. Phys., 117, 243

Moore, R. L. 1988, ApJ, 324, 1132

Moore, R. L., Falconer, D. A., Porter, J. G., \& Suess, S. T. 1999, ApJ, 526, 505 Moreton, G. E., \& Ramsey, H. E. 1960, PASP, 72, 357

Parnell, C. E. 2002, MNRAS, 335, 389

Porter, J. G., Moore, R. L., Reichmann, E. J., Engvold, O., \& Harvey, K. L. 1987, ApJ, 323, 380

Potts, H. E., Barrett, R. K., \& Diver, D. A. 2004, A\&A, 424, 253

Potts, H. E., Khan, J. I., \& Diver, D. A. 2007, Sol. Phys., 245, 55

Priest, E. R., Hood, A. W., \& Anzer, U. 1989, ApJ, 344, 1010

Priest, E. R., Parnell, C. E., \& Martin, S. F. 1994, ApJ, 427, 459

Priest, E. R., Heyvaerts, J. F., \& Title, A. M. 2002, ApJ, 576, 533

Schrijver, C. J., Title, A. M., van Ballegooijen, A. A., Hagenaar, H. J., \& Shine, R. A. 1997, ApJ, 487, 424

Thompson, B. J., Plunkett, S. P., Gurman, J. B., et al. 1998, Geophys. Res. Lett., 25,2465

van Ballegooijen, A. A., \& Martens, P. C. H. 1989, ApJ, 343, 971

van Ballegooijen, A. A., Priest, E. R., \& Mackay, D. H. 2000, ApJ, 539, 983

Wang, H. 1988, Sol. Phys., 116, 1

Wang, J., Li, W., Denker, C., et al. 2000, ApJ, 530, 1071

Warmuth, A., Vršnak, B., Magdalenić, J., Hanslmeier, A., \& Otruba, W. 2004, A\&A, 418, 1101 